\title{
The Effects Of Synbiotic Supplementation On Enteral Feeding Tolerance, Protein Homeostasis And Muscle Wasting Of Critically III Adult Patients: A Randomized Controlled Trial
}

\section{Najmeh Seifi}

Mashhad University of Medical Sciences

Reza Rezvani

Mashhad University of Medical Sciences

Alireza Sedaghat

Mashhad University of Medical Sciences

Mohsen Nematy

Mashhad University of Medical Sciences

Majid Khadem-rezaiyan

Mashhad University of Medical Sciences

Mohammad Safarian ( $\sim$ SafarianM@mums.ac.ir)

Mashhad University of Medical Sciences

\section{Research Article}

Keywords: Synbiotic, Critical IIIness, Enteral tolerance, Energy homeostasis, Nitrogen balance, Muscle wasting

Posted Date: March 3rd, 2022

DOl: https://doi.org/10.21203/rs.3.rs-779399/v1

License: (c) (1) This work is licensed under a Creative Commons Attribution 4.0 International License. Read Full License 


\section{Abstract}

Background: Enteral feeding intolerance, energy-protein malnutrition, and muscle wasting are common conditions in the critical care setting. The primary aim of this study was to investigate the effect of synbiotic supplementation on enteral feed volume, energy and protein homeostasis, and muscle mass maintenance in critically ill adult patients.

Methods: A consecutive of 42 patients admitted to the Edalatian Medical ICU, requiring enteral nutrition (EN) were prospectively randomized to receive the synbiotic capsule (containing a combination of Lactobacillus, Bifidobacterium, Streptococcus, and fructooligosaccharides) or placebo (21 patients in each group) for a maximum of 14 days.

Results: Mean EN volume, energy, and protein intake were $962.5 \pm 533.82 \mathrm{ml}, 770 \pm 427.05 \mathrm{kcal}$ and $38.5 \pm 21.35 \mathrm{~g}$ (fourth day) vs. $590 \pm 321.1 \mathrm{ml}, 472 \pm 256.81 \mathrm{kcal}$, and $23.6 \pm 12.84 \mathrm{~g}$ (first day) in the synbiotic group $(p<0.05)$. Changes in the placebo group were not statistically significant. On day 1 nitrogen balance (NB) was $-19.84 \pm 8.03$ in the synbiotic vs. $-10.99 \pm 9.12$ in the placebo group $(p=0.003)$. On day 14 , NB was $-14.18 \pm 13.05$ in the synbiotic and $-9.59 \pm 7.71$ in the placebo group $(p=0.41)$. Mid-arm circumference (MAC), 24-hour urine creatinine, and creatinine-height index were almost steady in the synbiotic group, while they decreased in the placebo group.

Conclusion: Overall, it can be concluded that enteral nutrition supplemented with synbiotics may increase enteral feed volume, energy and protein intake during the first four days. It is also associated with nitrogen balance improvement and muscle mass maintenance of critically ill adult patients.

Clinical Trial Registration Number: The trial protocol has been approved in Iranian Registry of Clinical Trials at 2019-03-17. The registration reference is IRCT20190227042857N1.

\section{Introduction}

The majority of patients in the intensive care unit (ICU) are admitted due to severe acute illness like sepsis, severe trauma, or major surgery. Therefore, cannot meet their nutritional needs accordingly, leading to energy-protein malnutrition and breakdown of muscle mass $(1,2)$.

Enteral feeding intolerance (EFI), a major contributor to malnutrition, is a common problem among ICU patients as it affects approximately one-third of ICU patients $(3,4)$. Several pathophysiological mechanisms contribute to EFI in critically ill patients, including alteration of hormonal and/or nervous system pathways, multiple drug delivery such as sedations and broad-spectrum antibiotics, inflammation, and biochemical disturbances (5).

Gut microbiota, the neglected endocrine organ, performs many crucial protective and metabolic functions which can affect enteral feeding tolerance(6). Gut microbiota can directly act on gastrointestinal (GI) smooth muscle contraction and mucosal absorption and secretion $(7,8)$. It can also modulate neuronal 
and hormonal pathways which ultimately regulate glucose homeostasis, appetite, and feeding performance $(9,10)$. It is also proposed that gut microbiota can influence protein balance and muscle mass maintenance independent of enteral tolerance and feed volume. Different theoretical mechanisms have been described for the gut-muscle axis which still needs to be identified $(11,12)$.

Considering the established extreme dysbiosis in critically ill patients (13) and the importance of malnutrition and muscle wasting in this setting, we aimed to evaluate the effect of gut microbiota modulation through synbiotics on enteral feeding tolerance, protein homeostasis, and muscle wasting of critically ill adult patients. To our knowledge, this is the first human clinical trial to evaluate the synbiotic supplementation effect on muscle wasting of critically ill patients.

\section{Materials And Methods}

This triple-blind randomized controlled clinical trial was conducted at the Edalatian intensive care unit of Imam Reza Hospital in Mashhad, Iran. The Research Ethics Committee of the School of Medicine at Mashhad University of Medical Sciences approved the study protocol (code:

IR.MUMS.MEDICAL.REC.1397.715), and the research has been registered at the Iranian Registry of Clinical Trials (No. IRCT20190227042857N1). The full protocol of this study is previously published (14). Here a brief description is provided.

\section{Inclusion and Exclusion Criteria}

ICU admitted, adult patients aged 18-65 years were included in the study if they or their guardian provided informed written consent and fulfilled the following criteria; having stable hemodynamics within 24-48 hours after admission; requiring enteral nutrition (EN) via a nasogastric tube (NGT); not receiving any microbial cell preparations (pre-, pro-, and synbiotic) during past 3 months.

Patients were excluded if they were pregnant and lactating; having any contraindications for EN or the placement of NGT, receiving immunosuppressive treatments, radiotherapy or chemotherapy and, or any of the following conditions: current cancer, or autoimmune diseases, known allergies to microbial cell preparations, transplants receiving, hematological diseases, acquired immunodeficiency syndrome, and congenital heart valve disease, or artificial heart valve.

Patients were also excluded from the final analysis if they have received the study intervention for less than 4 days.

\section{Product, Dosage, and Administration}

Initially, an online stratified sequential randomization was performed based on disease severity (APACHE II scores: 0-35 and 35-70). The researchers, health care staff, subjects and data analyzer were blinded to the procedures until the completion of the analysis. The secretary of the ICU ward was aware of randomization details. So, in case of any complications potentially attributed to the intervention, the medical staff could refer to her. 
Patients in both groups received hospital gavage through NGT every 3 hours (from 6 A.M. to the midnight). LactoCare synbiotic capsules (500 mg; Zist Takhmir, Iran) were administered to the synbiotic group every 12 hours. Each capsule contains Lactobacillus casei (1.5×109 CFU), Lactobacillus acidophilus (1.5×1010 CFU), Lactobacillus rhamnosus (3.5×109 CFU), Lactobacillus bulgaricus (2.5×108 $\mathrm{CFU})$, Bifidobacterium breve $(1 \times 1010 \mathrm{CFU})$, Bifidobacterium longum $(5 \times 108 \mathrm{CFU})$, Streptococcus thermophilus (1.5×108 CFU), and fructooligosaccharides. The capsule was diluted in five milliliters of water and administered via the NGT, separately via gavage after feeding. The patients in the control group received a placebo capsule (Zist Takhmir, Iran), which only contained sterile maize starch which was similar to the synbiotic capsules, even in the liquid form. The intervention was continued for a maximum of 14 days in both groups.

\section{Outcome Measurement}

\section{Nutritional risk}

To quantify the nutritional risk of critically ill patients we used the modified NUTRIC score. A score above 5 was considered as high risk.

\section{Enteral feed intolerance}

At each time of bullous delivery of hospital gavage, patients were evaluated for signs or symptoms of enteral intolerance. If there was vomiting, diarrhea, abdominal distention, or gastric residual volume (GRV) of more than $250 \mathrm{ml}$, feeding intolerance was recorded. The frequency of feeding intolerance records, the mean volume of received gavage, and prokinetic drug administration were considered as EFI. As the way enteral feed is delivered plays role in enteral feed tolerance, we educated the contributing nurses about bed angle and rate of administration before initiation of the study.

\section{Energy homeostasis}

Energy requirement was estimated based on the simple weight-based equation of $25 \mathrm{Kcal} / \mathrm{kg} / \mathrm{day}$. For day 1 , it was estimated that the patient needs to receive $30 \%$ of the estimated calorie. Each day we increased the estimated calorie requirement by $10 \%$. As our previous data showed that $1 \mathrm{ml}$ of our hospital gavage provides $0.8 \mathrm{kcal}$ of energy, we could calculate the total received calorie (Table 1 ). 
Table 1

Estimated and received energy calculations

\begin{tabular}{|lll|}
\hline Day & Estimated energy requirement $(\mathrm{kcal})$ & Received energy $(\mathrm{kcal})$ \\
\hline 1 & $(25 \mathrm{kcal} \times$ weight $) \times 30 \%$ & Received enteral volume $\times 0.8$ \\
\hline 2 & $(25 \mathrm{kcal} \times$ weight $) \times 40 \%$ & Received enteral volume $\times 0.8$ \\
\hline 3 & $(25 \mathrm{kcal} \times$ weight $) \times 50 \%$ & Received enteral volume $\times 0.8$ \\
\hline 4 & $(25 \mathrm{kcal} \times$ weight $) \times 60 \%$ & Received enteral volume $\times 0.8$ \\
\hline 5 & $(25 \mathrm{kcal} \times$ weight $) \times 70 \%$ & Received enteral volume $\times 0.8$ \\
\hline 7 & $(25 \mathrm{kcal} \times$ weight $) \times 80 \%$ & Received enteral volume $\times 0.8$ \\
\hline $8-14$ & $(25 \mathrm{kcal} \times$ weight $) \times 90 \%$ & Received enteral volume $\times 0.8$ \\
\hline
\end{tabular}

\section{Nitrogen balance (NB)}

NB was calculated by subtracting the total nitrogen $(\mathrm{N})$ output from the total $\mathrm{N}$ intake. Total $\mathrm{N}$ intake was determined by dividing total daily protein intake (gram) by 6.25 . The protein content of enteral gavage was $4 \mathrm{gr}$ per $100 \mathrm{ml}$. If patients received supplemental parenteral nutrition, parenteral protein intake was also calculated based on the amount of amino acids. A sample of 24-h urine collection was transferred to the laboratory to measure urinary urea nitrogen (UUN) by standard enzymatic method on days 1 and 14 . UUN plus 4 (skin and feces losses of $\mathrm{N}$ ) was subtracted from total $\mathrm{N}$ intake $(15,16)$.

$$
N B=[\text { totalproteinintake }(g r) \div 6.25]-(24 h U U N+4)
$$

\section{Muscle wasting}

To assess total body skeletal muscle, we used 24-h urine creatinine ( $\mathrm{Cr}$ ) excretion method $(17,18)$. 24-h urine collection was performed on days 1 and 14. Urine volume was recorded and an aliquot was instantly transferred to the laboratory to determine the Cr excretion by an autoanalyzer that used the Jaffe reaction. Based on previous evidence, to estimate muscle mass the following equilibrium was used (19).

$$
\operatorname{Musclemass}(K g)=[14.4 \times 24 \text { hurinaryCrexcretion }(g r)]+3.6
$$

As 24-h urine $\mathrm{Cr}$ is affected by anthropometric characteristics such as height, the 24-h urine $\mathrm{Cr} /$ height (CHI) was evaluated as an index of muscle and protein status(20).

Mid-arm circumference (MAC), the midway between acromion and olecranon processes of the left arm was measured 5 times during the study. All patients were lying with their arm beside.

\section{Statistical Analysis}


To achieve a statistical power of $80 \%$ with a two-sided significance level of 0.05 , we needed to enroll at least 36 patients. As we estimated a drop rate of $15 \%$, we registered 21 patients in each group of study.

Data analysis was performed in SPSS version 16 with an intent-to-treat principle. Independent and paired t-test, Mann-Whitney $U$ test or Wilcoxon test, and repeated measures for the quantitative data and Chisquare or Fisher's exact test for the qualitative data were applied. The reported P-values were two-tailed, and the P-value of less than 0.05 was considered statistically significant.

\section{Results}

Eligibility was determined in 280 ICU patients from April to October 2019, and 38 eligible patients were enrolled in the study (Fig. 1). Table 2 shows the demographic characteristics of the participants. No significant differences were observed in the baseline characteristics of the patients in the intervention and control groups. 
Table 2

Demographic and Baseline Characteristics of Patients

\begin{tabular}{|c|c|c|c|}
\hline & $\begin{array}{l}\text { Synbiotics } \\
(n=20)\end{array}$ & $\begin{array}{l}\text { Placebo } \\
(n=18)\end{array}$ & P-value \\
\hline Mean Age (year) & $38.5 \pm 17.94$ & $47.61 \pm 22.51$ & $0.35^{1}$ \\
\hline Gender N (\%) & & & $0.06^{2}$ \\
\hline Male & $9(45)$ & $14(78)$ & \\
\hline Female & $11(55)$ & $4(22)$ & \\
\hline \multicolumn{4}{|l|}{ Smoking Habits N (\%) } \\
\hline Previously & $10(50)$ & $12(66.7)$ & $0.29^{3}$ \\
\hline Currently & $9(45)$ & $10(55.5)$ & $0.52^{3}$ \\
\hline \multicolumn{4}{|l|}{ Past Medical History N (\%) } \\
\hline Diabetes & 0 & $3(16.6)$ & $0.09^{2}$ \\
\hline CVD & 0 & $3(16.6)$ & $0.09^{2}$ \\
\hline Neurological Disorders & $3(15)$ & $3(16.6)$ & $0.99^{2}$ \\
\hline Psychological Disorders & $5(25)$ & $4(22.2)$ & $0.99^{2}$ \\
\hline Cause of ICU Admission N (\%) & $12(60)$ & $10(55.5)$ & -4 \\
\hline Intoxication & $3(16.7)$ & $1(5)$ & \\
\hline Pulmonary & 0 & $3(15)$ & \\
\hline Cardiac & $1(5.6)$ & $1(5)$ & \\
\hline Neurologic & $3(16.7)$ & $2(10)$ & \\
\hline sepsis & $1(5.6)$ & $1(5)$ & \\
\hline \multicolumn{4}{|l|}{ others } \\
\hline Disease Severity (APACHEII) & $17.40 \pm 4.72$ & $18 \pm 4$ & $0.68^{5}$ \\
\hline Mean \pm SD & & & \\
\hline
\end{tabular}




\begin{tabular}{|l|l|}
\hline \multicolumn{1}{|c|}{$\begin{array}{c}\text { Synbiotics } \\
(\mathbf{n = 2 0})\end{array}$} & $\begin{array}{l}\text { Placebo } \\
(\mathbf{n = 1 8})\end{array}$ \\
\hline${ }^{1}$ Mann-Whitney U test; \\
${ }^{2}$ Fisher's exact test; \\
${ }^{3}$ Chi-square; \\
${ }^{4}$ Lack of Cochrane rules for chi-square testing; \\
${ }^{5}$ Independent t-test \\
\hline $\begin{array}{l}\text { CVD: cerebrovascular disorders; ICU: intensive care unit; APACHEII: Acute Physiology and Chronic } \\
\text { Health Evaluation }\end{array}$ \\
\hline
\end{tabular}

As it is shown in Table 3, the NUTRIC score on day 1, 7, and 14 was less than 5 (low nutritional risk) and there was no statistically significant difference between the two groups. EN start day, EN duration, and EN days per ICU stay days were similar in both groups. In the synbiotic group 3 patients (15\%) and in the placebo group 2 patients (11.1) needed supplemental PN during the study ( $p=0.99)$. Mean EN volume in the synbiotic and placebo groups was $1313.6 \pm 417.56 \mathrm{ml}$ and $1315.2 \pm 565.66 \mathrm{ml}$, respectively $(p=0.79)$. The mean energy deficit was about $300 \mathrm{kcal}$ in both groups ( $p=0.92)$. EFI was recorded 3.5 times in the synbiotic and 6.77 times in the placebo group. The difference was not statistically significant. Prokinetics administration frequency and duration were similar in both groups. 
Table 3

Nutritional status, enteral feed volume and energy homeostasis in the synbiotic and placebo groups

Synbiotics

$(n=20)$

\section{NUTRIC Score}

Day 1

Day 7

Day 14

EN start day

EN duration (days)

EN days per ICU stay days (\%)

Supplemental PN

$\mathrm{N}(\%)$

\section{Mean EN volume (ml)}

1 st week

$2^{\text {ed }}$ week

total

\section{Energy homeostasis (Kcal)}

1st week

$2^{\text {ed }}$ week

total

EFI records frequency

Prokinetics administration, $\mathrm{N}(\%)$

Prokinetics administration duration (days)

$2.85 \pm 1.89$
$2.7 \pm 1.4$

$2.42 \pm 2.02$

$3.08 \pm 2.06$

$8.85 \pm 4.41$

$93.52 \pm 12.67$

$3(15)$

$940.27 \pm 424.72$

$976.50 \pm 714.88$

$0.67^{2}$

$1507.7 \pm 495.85 \quad 1561.1 \pm 480.20$

$1313.6 \pm 417.56$

$1315.2 \pm 562.66$

$0.77^{2}$

$0.79^{2}$

P-value $(n=18)$ 
Synbiotics

$(n=20)$
Placebo

$(n=18)$

${ }^{1}$ Mann-Whitney U test;

2 Independent t-test;

${ }^{3}$ Fisher's exact test

EN, enteral nutrition; EFI, enteral feeding intolerance; ICU, intensive care unit; NUTRIC, nutrition risk in critically ill; N, number; $P N$, parenteral nutrition

As it is demonstrated in Table 4, protein intake during the first week, the second week, and all study duration was not significantly different between the two groups. Nitrogen balance was negative on days 1 and 14 in both study groups. On day 1 , it was -19.84 in the synbiotic and -10.99 in the placebo group $(p=0.003)$. On day 14 , it raised to -14.18 in the synbiotic and -9.59 in the placebo group $(p=0.41)$.

Table 4

Protein intake and nitrogen balance in the synbiotic and placebo groups

\begin{tabular}{|c|c|c|c|}
\hline & $\begin{array}{l}\text { Synbiotics } \\
(n=20)\end{array}$ & $\begin{array}{l}\text { Placebo } \\
(n=18)\end{array}$ & P-value \\
\hline \multicolumn{4}{|l|}{ Protein intake (g) } \\
\hline 1st week & $37.61 \pm 16.98$ & $39.06 \pm 28.59$ & $0.85^{1}$ \\
\hline $2^{\text {ed }}$ week & $60.3 \pm 19.83$ & $62.44 \pm 19.20$ & $0.79^{1}$ \\
\hline total & $52.54 \pm 16.7$ & $52.60 \pm 22.5$ & $0.99^{1}$ \\
\hline \multicolumn{4}{|c|}{ Protein intake per body weight $(\mathrm{g} / \mathrm{kg})$} \\
\hline 1st week & $0.78 \pm 0.2$ & $0.81 \pm 0.41$ & $0.82^{1}$ \\
\hline $2^{\text {ed }}$ week & $0.58 \pm 0.24$ & $0.62 \pm 0.48$ & $0.78^{1}$ \\
\hline total & $0.9 \pm 0.23$ & $0.96 \pm 0.37$ & $0.64^{1}$ \\
\hline Nitrogen balance (g) & $-19.84 \pm 8.03$ & $-10.99 \pm 9.12$ & $0.003^{2}$ \\
\hline $\begin{array}{l}\text { Day } 1 \\
\text { Day } 14\end{array}$ & $-14.18 \pm 13.05$ & $-9.59 \pm 7.71$ & $0.41^{2}$ \\
\hline \multicolumn{4}{|l|}{${ }^{1}$ Mann-Whitney U test; } \\
\hline
\end{tabular}

As Fig. 2 shows, enteral feed volume, calorie, and protein intake significantly increased from day 1 to day 4 in the synbiotic group. While the increase in the placebo group was not statistically significant. 
Mid-arm circumference measurements during the study period are presented in Fig. 3. In the placebo group, there was a significant linear reduction of MAC during the study $(p<0.001)$. But, in the synbiotic group changes were not statistically significant $(p=0.19)$.

As it is shown in Table 5, 24-h urine $\mathrm{Cr}$ and $\mathrm{CHI}$ decreased in the placebo group and was almost steady in the synbiotic group. Muscle mass also showed a $3 \mathrm{~kg}$ decrease in the placebo group, while it did not change in the synbiotic group. Although the differences are not statistically significant, they are clinically remarkable.

Table 5

Muscle mass status of synbiotic and placebo groups on day 1 and 14

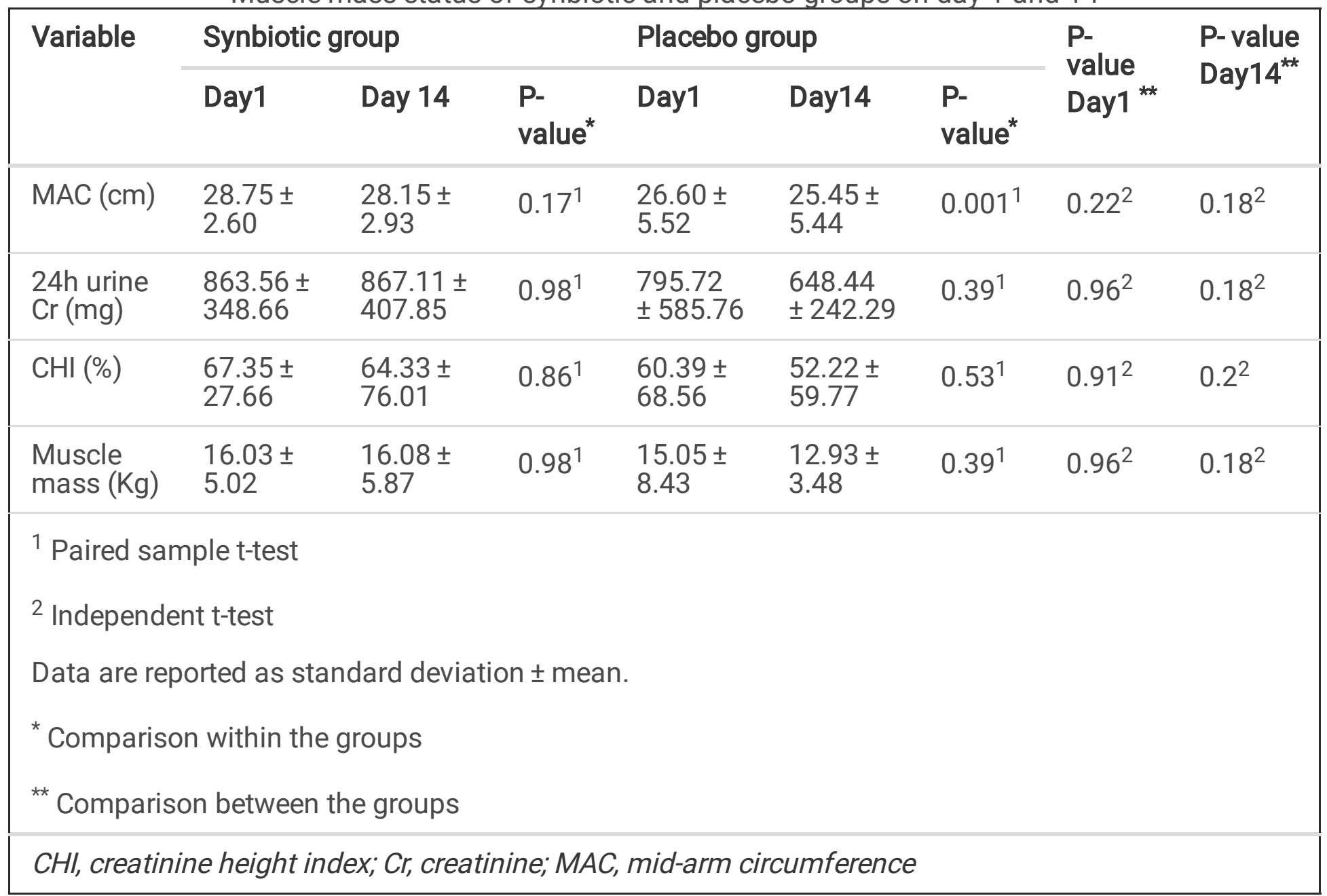

\section{Discussion}

In this study, we set out to investigate the effect of synbiotic supplementation on enteral feeding intolerance, protein status, and muscle maintenance of critically ill adult patients. The results of this study suggest that synbiotic are associated with a higher intake of hospital gavage, energy, and protein during the first four days, nitrogen balance improvement, and muscle mass maintenance in the synbiotic group. 
Although enteral feed volume, energy, and protein intake increased significantly during the first four days in the synbiotic group, the overall changes were not significant and there was no difference in the total EN volume and energy or protein intake between the two groups. Our recent systematic review also showed that synbiotic, probiotic or prebiotic does not affect energy intake and feed volume in tube-fed critically ill patients (21). Although, Rushdi et al showed that prebiotic supplementation was associated with significantly increased feed volume on day 4 in the intervention group (22), which was inconsistent with our results.

Delayed gastric emptying and feeding intolerance are common in the critical care setting(23). EFI usually occurs during the first few days after the start of EN (specifically on the $3^{\mathrm{d}}$ day). The underlying mechanisms are not well-known, but it seems that elevated level of CCK and PYY and their increased response to the presence of the small amount of nutrients in the gut, inflammation, hyperglycemia, variety of medications, as well as gut microbiota dysbiosis are among the involved mechanisms $(24,25)$. Dysbiosis occurs within a few hours of ICU admission (26) and its effect on feeding intolerance becomes evident during the first few days. Therefore, synbiotic supplementation efficacy through its effect on gut muscle contractions, secretion and absorption, glucose homeostasis and neuro-hormonal regulations, appetite, and feeding behavior $(7,10,11,27)$, is more related to the first few days of Enteral feeding.

Nitrogen balance was significantly more negative in the synbiotic group on day 1 . But, on day 14 , the difference between the two groups was not statistically significant. Although the improvement of nitrogen balance was not statistically significant in either group, it was clinically remarkable in the synbiotic group. As energy and protein intake were similar in the two groups, the improvement may be attributed to the improvement of digestion and absorption of proteins in the GI tract. Some previous studies showed that probiotics can affect digestion and absorption of proteins through different mechanisms including activation of digestive protease and peptidase, improvement of absorption of small peptides and aminoamides, reduction of harmful protein fermentation. In contrast to our results, Falco de Arruda et al. showed that enteral nutrition supplemented with probiotics and glutamine was not associated with nitrogen balance improvement in head trauma patients (28).

The results of this study showed that MAC in the synbiotic group remained almost constant during the study, while it significantly decreased in the placebo group. 24-hour urine $\mathrm{Cr}$ and $\mathrm{CHI}$, as indicators of muscle mass, also remained constant in the synbiotic group, while they decreased in the placebo group. Although this decrease was not statistically significant, the reduction of $2 \mathrm{~kg}$ of muscle mass in the placebo group seems to be clinically remarkable. Numerous animal studies support the effect of gut microbiota homeostasis on muscle mass maintenance (29-32), but no human study have examined this effect. According to the limited available studies, it seems that gut microbiota affects muscle pathophysiology through different mechanisms including maintenance of gut barrier function, reduction of endotoxin translocation and inflammation, improvement of insulin sensitivity, mitochondrial biogenesis, muscle anabolism, and reduction of myocyte apoptosis by important metabolites such as short- chain fatty acids (SCFAs), regulation of amino acids bioavailability for muscle protein synthesis, 
anabolism stimulation and oxidative stress suppression by synthesis of Vit B group, glycine, and betaine $(33,34)$.

To our knowledge, this is the first human clinical trial investigating the effect of synbiotic supplementation on muscle wasting in critically ill patients. Although, there are some limitations. We feed our patients with hospital gavage which contains meat protein. If we could use standard commercial gavage, we could also measure 24-hours urine 3-methyl- Histidine $(3 \mathrm{MH})$, which is exclusively found in the skeletal muscle, and its urinary excretion shows muscle protein degradation (35). Also, due to executive restrictions, we were not able to increase the enteral feed volume to the patients' tolerable level and we obeyed the enteral nutrition policy of the ICU ward. Although their policy is to gradually increase EN in case of tolerance and decrease in case of intolerance, our results cannot lead to an exact conclusion about the enteral feed tolerance of our patients. Finally, we suggest further clinical trials that determine gut microbiota changes by molecular techniques.

\title{
Conclusion
}

The results of this study demonstrated that enteral nutrition supplemented with synbiotics containing a combination of Lactobacillus, Bifidobacterium, Streptococcus, and fructooligosaccharides, can increase enteral feed volume, and energy and protein intake during the first four days. It is also associated with nitrogen balance improvement and muscle mass maintenance of critically ill adult patients.

\section{Abbreviations}

\author{
A.M.: Ante Meridiem
}

APACHE II: Acute Physiology and Chronic Health Evaluation

CCK: Cholecystokinin

CFU: Colony-Forming Unit

CHI: Height Index

Cr: Creatinine

CVD: Cerebrovascular Disorders

EFI: Enteral Feeding Intolerance

EN: Enteral Nutrition

GI: Gastrointestinal

GRV: Gastric Residual Volume 
ICU: Intensive Care Unit

MAC: Mid-Arm Circumference

$\mathrm{N}$ : Nitrogen

NB: Nitrogen Balance

NGT: Nasogastric Tube

NUTRIC: Nutrition Risk in Critically III

PN: Parenteral Nutrition

PYY: Peptide YY

SCFA: Short- Chain Fatty Acids

UUN: Urinary Urea Nitrogen

3MH: 3-Methyl- Histidine

\section{Declarations}

- Ethics approval and consent to participate:

Ethical approval was obtained from ethical committee of MUMS. The ethical approval code is IR.MUMS.MEDICAL.REC.1397.715. The informed consent was obtained from all study participants or their legal guardian.

\section{- Consent for publication}

No personal identifying information was published.

- Availability of data and materials

The datasets generated and/or analyzed during the current study are not publicly available due to ethical considerations, but may be available from the corresponding author on reasonable request.

- Conflicts of interest:

None declared.

- Funding:

This research was funded by the Vice Chancellor for Research of MUMS (No. 971047). 


\section{- Authors' contributions:}

N. Seifi, M. Safarian and R.Rezvani equally contributed to the conception and design of the research; M. Nematy contributed to the design of the research; N. Seifi contributed to the acquisition and analysis of the data; M. Khadem-Rezaiyan and A. Sedaghat contributed to the interpretation of the data; and N. Seifi drafted the manuscript. All authors critically revised the manuscript, agree to be fully accountable for ensuring the integrity and accuracy of the work, and read and approved the final manuscript.

\section{- Acknowledgements:}

Hereby, we would like to show our gratitude to Mashhad University of Medical Sciences (MUMS) for helping in this research project. We are also immensely grateful to the Clinical Research Development Unit of Akbar Hospital for the study design and statistical analysis of the data. This article was extracted from a Ph.D. thesis by Dr. Najmeh Seifi (thesis number: A-1492).

\section{References}

1. Quirk J. Malnutrition in critically ill patients in internsive care units. British journal of nursing. 2000;9(9):537-41.

2. Cederholm T, Bosaeus I, Barazzoni R, Bauer J, Van Gossum A, Klek S, et al. Diagnostic criteria for malnutrition-an ESPEN consensus statement. Clinical nutrition. 2015;34(3):335-40.

3. McClave SA, Gualdoni J, Nagengast A, Marsano LS, Bandy K, Martindale RG. Gastrointestinal dysfunction and feeding intolerance in critical illness: do we need an objective scoring system? Curr Gastroenterol Rep. 2020;22(1):1-8.

4. Blaser AR, Starkopf J, Kirsimägi Ü, Deane A. Definition, prevalence, and outcome of feeding intolerance in intensive care: a systematic review and meta-analysis. Acta Anaesthesiol Scand. 2014;58(8):914-22.

5. Reintam Blaser A, Deane AM, Preiser JC, Arabi YM, Jakob SM. Enteral feeding intolerance: updates in definitions and pathophysiology. Nutrition in clinical practice. 2021;36(1):40-9.

6. Clarke G, Stilling RM, Kennedy PJ, Stanton C, Cryan JF, Dinan TG. Minireview: gut microbiota: the neglected endocrine organ. Molecular endocrinology. 2014;28(8):1221-38.

7. Verdu EJN. Motility. Probiotics effects on gastrointestinal function: beyond the gut? 2009;21(5):477 -80 .

8. Distrutti E, Monaldi L, Ricci P, Fiorucci, SJWjog. Gut microbiota role in irritable bowel syndrome. New therapeutic strategies. 2016;22(7):2219.

9. Cani PD, Geurts L, Matamoros S, Plovier H, Duparc TJD, metabolism. Glucose metabolism: focus on gut microbiota, the endocannabinoid system and beyond. 2014;40(4):246 - 57.

10. Spiljar M, Merkler D, Trajkovski MJFii. The immune system bridges the gut microbiota with systemic energy homeostasis: focus on TLRs, mucosal barrier, and SCFAs. 2017;8:1353. 
11. Wang J, Ji H. Influence of Probiotics on Dietary Protein Digestion and Utilization in the Gastrointestinal Tract. Curr Protein Pept Sci. 2019;20(2):125-31.

12. Ticinesi A, Lauretani F, Milani C, Nouvenne A, Tana C, Del Rio D, et al. Aging gut microbiota at the cross-road between nutrition, physical frailty, and sarcopenia: is there a gut-muscle axis? 2017;9(12):1303.

13. McDonald D, Ackermann G, Khailova L, Baird C, Heyland D, Kozar R, et al. Extreme dysbiosis of the microbiome in critical illness. Msphere. 2016;1(4):e00199-16.

14. Seifi N, Safarian M, Nematy M, Rezvani R, Khadem-Rezaian M, Sedaghat A. Effects of synbiotic supplementation on energy and macronutrients homeostasis and muscle wasting of critical care patients: study protocol and a review of previous studies. Trials. 2020;21(1):1-11.

15. Pupim L, Martin C, Ikizler T. Assessment of protein and energy nutritional status. Nutritional Management of Renal Disease. Academic Press; 2013.

16. Dickerson RN. Nitrogen balance and protein requirements for critically ill older patients. Nutrients. 2016;8(4):226.

17. Oterdoom LH, Gansevoort RT, Schouten JP, de Jong PE, Gans RO, Bakker SJ. Urinary creatinine excretion, an indirect measure of muscle mass, is an independent predictor of cardiovascular disease and mortality in the general population. Atherosclerosis. 2009;207(2):534-40.

18. Heymsfield SB, Arteaga C, McManus C, Smith J, Moffitt S. Measurement of muscle mass in humans: validity of the 24-hour urinary creatinine method. Am J Clin Nutr. 1983;37(3):478-94.

19. Wang Z, Gallagher D, Nelson ME, Matthews DE, Heymsfield SB. Total-body skeletal muscle mass: evaluation of 24-h urinary creatinine excretion by computerized axial tomography. Am J Clin Nutr. $1996 ; 63(6): 863-9$.

20. Walser M. Creatinine excretion as a measure of protein nutrition in adults of varying age. Journal of Parenteral Enteral Nutrition. 1987;11:73S-8S.

21. Seifi N, Esfahani AJ, Sedaghat A, Rezvani R, Khadem-Rezaiyan M, Nematy M, et al. Effect of gut microbiota modulation on feeding tolerance of enterally fed critically ill adult patients: a systematic review. Systematic reviews. 2021;10(1):1-11.

22. Rushdi TA, Pichard C, Khater YH. Control of diarrhea by fiber-enriched diet in ICU patients on enteral nutrition: a prospective randomized controlled trial. Clin Nutr. 2004;23(6):1344-52.

23. Tempest M. Enteral nutrition intolerance in critical illness. Journal of Parenteral Enteral Nutrition. $2011 ; 13(2): 30$.

24. Deane A, Chapman MJ, Fraser RJ, Bryant LK, Burgstad C, Nguyen NQJWjogW. Mechanisms underlying feed intolerance in the critically ill: implications for treatment. 2007;13(29):3909.

25. Chen W-T, Du M-J, Chen Y-Z, Yuan D-QJIJCEM. Factors influencing feeding intolerance in critically ill patients during enteral nutrition. 2019;12(7):7999-8003.

26. Alverdy JC, Krezalek MAJCcm. Collapse of the microbiome, emergence of the pathobiome and the immunopathology of sepsis. 2017;45(2):337. 
27. Cani PD, Geurts L, Matamoros S, Plovier H, Duparc T. Glucose metabolism: focus on gut microbiota, the endocannabinoid system and beyond. Diabetes Metab. 2014;40(4):246-57.

28. Falcao de Arruda IS, de Aguilar-Nascimento JE. Benefits of early enteral nutrition with glutamine and probiotics in brain injury patients. Clin Sci. 2004;106(3):287-92.

29. Varian BJ, Goureshetti S, Poutahidis T, Lakritz JR, Levkovich T, Kwok C, et al. Beneficial bacteria inhibit cachexia. Oncotarget. 2016;7(11):11803.

30. Bindels LB, Beck R, Schakman O, Martin JC, De Backer F, Sohet FM, et al. Restoring specific lactobacilli levels decreases inflammation and muscle atrophy markers in an acute leukemia mouse model. PloS one. 2012;7(6):e37971.

31. Bindels LB, Neyrinck AM, Claus SP, Le Roy Cl, Grangette C, Pot B, et al. Synbiotic approach restores intestinal homeostasis and prolongs survival in leukaemic mice with cachexia. ISME J. 2016;10(6):1456-70.

32. Bindels LB, Neyrinck AM, Salazar N, Taminiau B, Druart C, Muccioli GG, et al. Non digestible oligosaccharides modulate the gut microbiota to control the development of leukemia and associated cachexia in mice. PloS one. 2015;10(6):e0131009.

33. Ticinesi A, Lauretani F, Milani C, Nouvenne A, Tana C, Del Rio D, et al. Aging gut microbiota at the cross-road between nutrition, physical frailty, and sarcopenia: is there a gut-muscle axis? Nutrients. 2017;9(12):1303.

34. Dukes A, Davis C, El Refaey M, Upadhyay S, Mork S, Arounleut P, et al. The aromatic amino acid tryptophan stimulates skeletal muscle IGF1/p70s6k/mTor signaling in vivo and the expression of myogenic genes in vitro. Nutrition. 2015;31(7-8):1018-24.

35. Elia M, Carter A, Bacon S, Winearls C, Smith R. Clinical usefulness of urinary 3-methylhistidine excretion in indicating muscle protein breakdown. Br Med J (Clin Res Ed). 1981;282(6261):351-4.

\section{Figures}

\section{Figure 1}

CONSORT flow diagram

\section{Figure 2}

Enteral feed volume, energy and protein intake on day 1 and 4 in the synbiotic and placebo groups 
Figure 3

Mid-arm circumference changes during the study period 